\title{
Establishment and Simulation of the Articular Cartilage Two Phase Model Based on the Hyperelastic Solid Phase
}

\author{
Monan Wang ${ }^{1 *}$, Qi Wang ${ }^{2}$ and Jianan Liang ${ }^{3}$ \\ Mechanical \& Power Engineering College, Harbin University of Science and \\ Technology, Harbin, China \\ 1qqwmnan@163.com, ${ }^{2} 253171357 @ q q . c o m,{ }^{3} 561295016 @ q q . c o m$
}

\begin{abstract}
Articular cartilage is composed by fiber reinforced solid phase and free flowing liquid phase, the study aimed to establish an articular cartilage two phase model with respect to the structural characteristics of articular cartilage. According to the Helmholtz strain energy function, the hyperelastic property of solid phases is defined, the liquid phase is defined as an ideal fluid. The model can describe the non-linearity, incompressibility and permeability of articular cartilage. The balance equation of the finite element based on the v-p variable was obtained by the Galerkin weighted residual approach, using finite difference method to calculate the equilibrium equation, the computation program was compiled in Microsoft Visual Studio 2012. Finally, the stress and strain data obtained from the simulation of the articular cartilage model were compared with the corresponding experimental data, the results show that the two phase model based on the hyperelastic solid phase can meet the accuracy requirements of the clinical application of cartilage model.
\end{abstract}

Keywords: articular cartilage; two phase model; nonlinear; hyperelastic solid phase; finite element method

\section{Introduction}

Articular cartilage is composed of water and complex organic matter. Complex organic matter mainly includes collagen and proteoglycan. Interstitial fluid contains mostly water, the flow of the fluid not only has an important influence on cartilage mechanical properties, also has a close relationship with the vascular tissue nutrient transport [1]. At the same time, articular cartilage has a very low coefficient of friction, which plays a good role in lubrication [2]. The lubrication effect of articular cartilage has a very important significance for the ability of dynamic joint movement and bearing load. Therefore, studying on the mechanical properties of articular cartilage is very important for the development of medical research, clinical diagnosis and new biological material $[3,4]$. The finite element method was used to study the biomechanics of orthopedics, the numerical simulation method was also used to analyze the complex mechanical behavior of articular cartilage [5].

In the early stage of articular cartilage mechanical modeling study, the cartilage was considered as isotropic linear elastic single-phase material. In 1976, Hori and Mockros regarded cartilage as viscoelastic material and remeasured shear modulus [6]. Hayes considered cartilage as a homogeneous isotropic material, and a generalized Kelvin model was established, and the shear modulus was obtained [7]. During this time, the researchers accept the viscoelastic single-phase articular cartilage model, however, the simulation results are always different with the true cartilage properties and the experimental data. In 1980, Mow and Lai proposed a two - phase medium model based on mixture theory,

* Corresponding Author 
which regarded cartilage as an incompressible mixture consisting of linear elastic solid and non-viscous liquid [8,9], Mow got the experimental data obtained by the compression experiment, which was very close to the simulation results, the model is widely used in next 20 years. But, this model still has some limitations, the theory of viscoelasticity was developed by Li and Armstrong, the viscoelastic behavior of cartilage was described by a two phase model, and the conditions of creep and stress relaxation were also presented. In 1999, the performance of cartilage was studied by means of finite element method, by Yan Bo Chongqing University [10]. In these studies, the solid phase of cartilage is also considered as linear elastic material, and the linear elastic model is only effective for small deformation. Aiming at the problem that the articular cartilage in the process of bearing load can produce large deformation, the finite deformation two order model of solid phase was established by Mow and Holmes [11].

In this study, articular cartilage was described as a two phase mixture consisting of incompressible non-viscous liquid phase and hyperelastic, transversely isotropic solid phase, and under the condition of deformation, the permeability of cartilage tissue can be changed. The stress-strain relationship of solid phase was defined by the Helmholtz strain energy function. The balance equation of finite element system based on $v-p$ variable is obtained by Galerkin weighted residual method, finite difference method is used to solve, the finite element analysis program is compiled in Microsoft Visual Studio 2012.

\section{The Establishment of the Control Equation of the Articular Cartilage Two Phase Model Based on the Mixture Theory}

\subsection{Establishment of Two Phase Model of Articular Cartilage}

In this study, articular cartilage is regarded as a two phase medium mixture, which is composed of a hyperelastic solid phase and an ideal fluid phase, and each phase has its own movement rules. The solid phase is expressed by $\mathrm{s}$, and the liquid phase is expressed by $\mathrm{f}$. The initial volume fraction of each phase is $\phi_{\mathrm{s} 0}, \phi_{\mathrm{f} 0}$, the initial density of each phase is $\rho_{\mathrm{s} 0}^{\mathrm{r}}, \rho_{\mathrm{f} 0}^{\mathrm{r}}$, and uniformly distributed.

The formula of the volume fraction under saturated state as follows:

$$
\phi_{\mathrm{s}}+\phi_{\mathrm{f}}=1
$$

Mass balance equation is as follow:

$$
\nabla \cdot\left(\phi_{\mathrm{s}} v_{\mathrm{s}}+\phi_{\mathrm{f}} v_{\mathrm{f}}\right)=0
$$

In the quasi-static problem, the momentum balance equation is transformed into the static equilibrium equation, and the acceleration $\boldsymbol{a}$ is zero. In the two-phase mixture, the momentum exchange formed by the friction resistance which is brought when the interstitial fluid flows through the porous solid is $P_{\mathrm{s}}$ and $P_{\mathrm{f}}$, the relationship between them is as follow:

$$
P_{\mathrm{s}}=-P_{\mathrm{f}}=K\left(v_{\mathrm{f}}-v_{\mathrm{s}}\right)
$$

In the formula, $K$ is the diffusion resistance coefficient.

$$
K=\frac{\left(\phi_{\mathrm{r}}\right)^{2}}{\kappa}
$$

$\boldsymbol{\sigma}_{\mathrm{s}}$ and $\boldsymbol{\sigma}_{\mathrm{f}}$ are solid phase Cauchy stress tensor and liquid phase Cauchy stress tensor respectively, the momentum balance equation of the cartilage in the current configuration is: 


$$
\begin{gathered}
\nabla \cdot \boldsymbol{\sigma}_{\mathrm{s}}+P_{\mathrm{s}}=0 \\
\nabla \cdot \boldsymbol{\sigma}_{\mathrm{f}}+P_{\mathrm{f}}=0
\end{gathered}
$$

The mechanical constitutive equations of elastic solid and non-viscous fluid are obtained by the incompressibility and mechanical properties of cartilage:

$$
\begin{aligned}
& \sigma_{\mathrm{s}}=-\phi_{\mathrm{s}} p \mathbf{I}+\sigma_{\mathrm{e}} \\
& \sigma_{\mathrm{f}}=-\phi_{\mathrm{f}} p \mathbf{I}
\end{aligned}
$$

$p$ is the pressure, $\boldsymbol{\sigma}_{\mathbf{e}}$ is the effective stress of the solid phase.

The mass balance equation (2), momentum balance equation (5)(6) and constitutive equation of two phase mixture (7)(8) are obtained by the above deduction, thus the governing equations for the mechanical analysis of articular cartilage are formed.

The relationship between the second Piola-Kirchhoff stress tensor and elastic stress tensor is:

$$
\boldsymbol{\sigma}_{\mathrm{e}}=\boldsymbol{J}^{-1} \mathbf{F S F}^{T}=\mathbf{F S F}^{T}
$$

The $\mathbf{S}$ is second Piola-Kirchhoff stress tensor. The parameter is solved in 2.3, and the hyper elastic two-phase model of articular cartilage can be obtained through the connection of the parameters.

In the deformation, the change of object space position before and after bearing load is considered, when $t=0$, the space region $V_{0}$ occupied by the object is the initial configuration, at the current time $t$, the spatial region $V$ occupied by the object is the current configuration.

The initial configuration is the reference configuration, can get the change of volume element and surface element in deformation, therefore, volume of the element in the current configuration and the volume in reference configuration have the following relationship.

$$
d V=J d V_{0}
$$

Articular cartilage is regarded as incompressible material, i.e., the density of material has no change before and after deformation, therefore, the area of element in the current configuration and the area in reference configuration have the following relationship.

$$
n d \Gamma=J n_{0} \mathbf{F}^{-1} d \Gamma_{0}
$$

$\Gamma$ represents the area of boundary region at current time, $\Gamma_{0}$ represents the area of the boundary region at the initial moment, $n$ represents the unit normal vector of the boundary area in the current configuration. $n_{0}$ represents the unit normal vector of the boundary area in the initial configuration.

By using the right Cauchy-Green strain tensor $\mathbf{C}$, the above formula can also be expressed as follows:

$$
\begin{gathered}
d \Gamma=J_{\Gamma} d \Gamma_{0} \\
J_{\Gamma}=J \sqrt{n_{0} C^{-1} n_{0}}=\sqrt{n_{0} C^{-1} n_{0}}
\end{gathered}
$$




\subsection{The Establishment of Hyperelastic Model}

The hyperelastic model of articular cartilage is represented by the Helmholtz strain energy function [12], as follows:

$$
\Psi=\alpha_{0} \frac{e^{\alpha_{1}\left(I_{1}-3\right)+\alpha_{2}\left(I_{2}-3\right)}}{I_{3}^{\beta}}
$$

\subsection{The Solution of Second Piola-Kirchhoff Stress Tensor S}

Hyperelastic solid phase of articular cartilage, the relationship between stress and strain is no longer linear, the elastic matrix is related to the strain tensor, The constitutive relation of stress and strain tensor of the second Piola-Kirchhoff stress $\mathbf{S}$ and Green strain $\mathbf{E}$, in an instantaneous deformation process, is: $d \mathbf{S}=\mathbf{D} d \mathbf{E}$.

Green strain tensor $\mathbf{E}$ is:

$$
\mathbf{E}=\left[\begin{array}{lll}
E_{11} & E_{12} & E_{13} \\
E_{21} & E_{22} & E_{23} \\
E_{31} & E_{32} & E_{33}
\end{array}\right]
$$

$\mathbf{S}=\frac{\partial \Psi}{\partial \mathbf{E}}=$

$$
\Psi\left[\begin{array}{ccc}
2 \alpha_{1}+4 \alpha_{2}\left(1+E_{22}+E_{33}\right) & -8 \alpha_{2} E_{12} & -8 \alpha_{2} E_{13} \\
-8 \alpha_{2} E_{12} & 2 \alpha_{1}+4 \alpha_{2}\left(1+E_{11}+E_{33}\right) & -8 \alpha_{2} E_{23} \\
-8 \alpha_{2} E_{13} & -8 \alpha_{2} E_{23} & 2 \alpha_{1}+4 \alpha_{2}\left(1+E_{11}+E_{22}\right)
\end{array}\right]
$$

Elastic tensor D can be expressed as:

$$
\begin{aligned}
& D_{i j k l}=\frac{\partial S_{i j}}{\partial E_{k l}}=\left[\begin{array}{cccccc}
D_{1111} & D_{1122} & D_{1133} & D_{1123} & D_{1113} & D_{1112} \\
D_{2211} & D_{2222} & D_{2233} & D_{2223} & D_{2213} & D_{2212} \\
D_{3311} & D_{3322} & D_{3333} & D_{3323} & D_{3313} & D_{3312} \\
D_{2311} & D_{2322} & D_{2333} & D_{2323} & D_{2313} & D_{2312} \\
D_{1311} & D_{1322} & D_{1333} & D_{1323} & D_{1313} & D_{1312} \\
D_{1211} & D_{1222} & D_{1233} & D_{1223} & D_{1213} & D_{1212}
\end{array}\right] \\
& 2 \alpha_{1}+4 \alpha_{2}\left(1+E_{22}+E_{33}\right)=G \\
& 2 \alpha_{1}+4 \alpha_{2}\left(1+E_{11}+E_{22}\right)=H \\
& 2 \alpha_{1}+4 \alpha_{2}\left(1+E_{11}+E_{33}\right)=J
\end{aligned}
$$

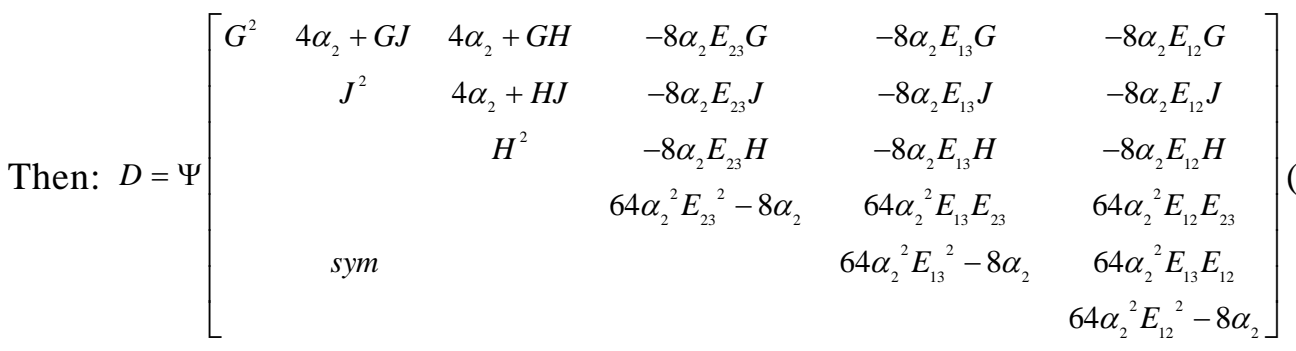

Articular cartilage can also cause the change of tissue permeability under the condition of deformation. By cartilage quasi incompressibility knows that $I_{3}=1$, 
cartilage permeability is expressed as $\kappa=\kappa_{0}\left(\frac{\phi_{s 0} \phi_{s}}{\phi_{f 0} \phi_{f}}\right)^{L}$. Where $L$ is constant, the subscript " 0 " represents the initial configuration before the deformation.

\subsection{The Establishment of Control Equation Based on $v-p$ Variable}

In this study, the velocity and pressure are selected to establish the $v-p$ variable finite element equation, solving the two phase model of articular cartilage. In order to eliminate the velocity of the fluid phase, first, the formula (3) and the formula (8) are brought to the formula (6), it can obtained by formula (4):

$$
\phi_{\mathrm{f}}\left(v_{\mathrm{f}}-v_{\mathrm{s}}\right)=-\kappa \nabla p
$$

Under the condition of formula (1), formula (20) is brought into the formula (2), the equation which contain solid phase velocity is obtained:

$$
\nabla \cdot\left(v_{\mathrm{s}}-\kappa \nabla p\right)=0
$$

Simultaneous momentum conservation equations (5) (6) and constitutive equations (7) (8) can get that:

$$
\nabla \cdot\left(\boldsymbol{\sigma}_{\mathrm{e}}-p \mathbf{I}\right)=0
$$

Equation (21) and Equation (22) constitute the finite element control equation that based on $v-p$ variable.

\section{Establishment and Simulation of the Articular Cartilage Mechanical Equilibrium Equation}

\subsection{Boundary Conditions}

Fluid flux is defined as follows: $\bar{Q}=\phi_{\mathrm{f}}\left(v_{\mathrm{f}}-v_{\mathrm{s}}\right) \cdot n=-\kappa \nabla p \cdot n$, The control conditions of the governing equations are:

$$
\begin{gathered}
u_{\mathrm{s}}(x, t)=\bar{u}_{\mathrm{s}}, x \in \Gamma_{\mathrm{u}_{\mathrm{s}}} \\
\sigma(x, t) \cdot n=\bar{T}, x \in \Gamma_{T} \\
p(x, t)=\bar{p}, x \in \Gamma_{p} \\
-\kappa \nabla p \cdot n=\bar{Q}, x \in \Gamma_{Q}
\end{gathered}
$$

Where $\bar{u}_{\mathrm{s}}$ is a known solid phase displacement, $\bar{T}$ is the total load force, $\bar{p}$ is known pressure, $n$ is the outer normal vector of the boundary $\Gamma, \Gamma_{\mathrm{p}}$ and $\Gamma_{\mathrm{Q}}$ represent the boundary of liquid phase volume domain $\Omega_{\mathrm{f}}$, respectively, $\Gamma_{\mathrm{u}_{\mathrm{s}}}$ and $\Gamma_{\mathrm{T}}$ respectively represent the boundary of the solid phase volume domain $\mathrm{C}$, and the boundary satisfies the following conditions:

$$
\begin{gathered}
\Gamma_{\mathrm{u}_{\mathrm{s}}} \cup \Gamma_{\mathrm{T}}=\Gamma_{\mathrm{s}}, \quad \Gamma_{\mathrm{u}_{\mathrm{s}}} \cap \Gamma_{\mathrm{T}}=\varnothing \\
\Gamma_{\mathrm{p}} \cup \Gamma_{\mathrm{Q}}=\Gamma_{\mathrm{f}}, \quad \Gamma_{\mathrm{p}} \cap \Gamma_{\mathrm{Q}}=\varnothing
\end{gathered}
$$




\subsection{The Establishment of System Equilibrium Equation}

Mesh types in this study are tetrahedral elements. The interpolation function of the tetrahedral element is constructed. By means of the governing equations based on the $v-p$ variables and boundary conditions, the finite element equilibrium equation is established by using Galerkin weighted residual method. Select the shape function as the weighting function of differential equations and boundary conditions, weight functions $\mathrm{N}_{\mathrm{J}}$ and $-\mathrm{N}_{J}$ are introduced, by the Gauss divergence theorem and the definition of the total traction force, the simplified weighted residual integral expression is obtained:

$$
\begin{aligned}
& \int_{\Omega}\left\{\nabla \mathbf{N}_{\mathrm{J}}^{T}: \mathbf{\sigma}_{\mathrm{e}}-\mathbf{N}_{\mathrm{J}}^{T} \nabla \cdot v_{\mathrm{s}}-p \nabla \cdot \mathbf{N}_{\mathrm{J}}^{T}-\kappa\left(\nabla p \cdot \nabla \mathbf{N}_{\mathrm{J}}^{T}\right)\right\} d \Omega \\
& =\int_{\Gamma_{T}} \mathbf{N}_{\mathrm{J}}^{T} \cdot \bar{T} d \Gamma+\int_{\Gamma_{Q}} \mathbf{N}_{\mathrm{J}}^{T} \cdot \bar{Q} d \Gamma
\end{aligned}
$$

The problem domain is discretized into the form of the element, and the velocity and pressure are interpolated:

$$
\begin{gathered}
v=\mathbf{N} v_{\mathrm{e}} \\
p=\mathbf{N} p_{\mathrm{e}}
\end{gathered}
$$

The matrix form of the weighted residual expression on a grid in the reference configuration is:

$$
\left[\begin{array}{ll}
\mathbf{e}_{\mathrm{n}} & \mathbf{e}_{\mathrm{n}}
\end{array}\right]\left\{\left[\begin{array}{cc}
0 & G_{\mathrm{e}}^{T} \\
G_{\mathrm{e}} & H_{\mathrm{e}}
\end{array}\right]\left[\begin{array}{c}
v_{\mathrm{e}} \\
p_{\mathrm{e}}
\end{array}\right]+\left[\begin{array}{c}
M_{\mathrm{e}}^{\mathrm{s}} \\
0
\end{array}\right]\right\}=\left[\begin{array}{ll}
\mathbf{e}_{\mathrm{n}} & \mathbf{e}_{\mathrm{n}}
\end{array}\right]\left[\begin{array}{l}
T \\
Q
\end{array}\right]
$$

Where, $\mathbf{e}_{\mathrm{n}}$ is a unit vector, because $\mathbf{e}_{\mathrm{n}}$ is a nonzero constant vector, so it can directly eliminate.

We supposed $G_{\mathrm{e}}=\int_{\Omega_{0}}-\mathbf{N}_{\jmath}^{T} \nabla \cdot \mathbf{N} d \Omega_{0}, \quad G_{\mathrm{e}}^{T}=\int_{\Omega_{0}}-(\nabla \cdot \mathbf{N}) \mathbf{N}_{J}^{T} d \Omega_{0}, \quad H_{\mathrm{e}}=\int_{\Omega_{0}}-\nabla \cdot \mathbf{N}_{\mathrm{J}}^{T} k \nabla \cdot \mathbf{N} d \Omega_{0}, \quad M_{\mathrm{e}}^{\mathrm{s}}=\int_{\Omega_{0}} t r\left[\left(\nabla_{0} \mathbf{N}_{\mathrm{J}}\right)^{T} \mathbf{F s}\right] d \Omega_{0}$, $T=\int_{\Gamma_{T_{0}}} \mathbf{N}_{J}^{T} \cdot \bar{T}_{J_{\Gamma}} d \Gamma_{0}, \quad Q=\int_{\Gamma_{Q_{0}}} \mathbf{N}_{J}^{T} \cdot \bar{Q}_{\Gamma} d \Gamma_{0}$, Then:

$$
\mathbf{Y}=\left[\begin{array}{cc}
0 & \mathbf{G}_{\mathrm{e}}{ }^{T} \\
\mathbf{G}_{\mathrm{e}} & \mathbf{H}_{\mathrm{e}}
\end{array}\right], \mathbf{v}=\left[\begin{array}{c}
v_{\mathrm{e}} \\
p_{\mathrm{e}}
\end{array}\right], \mathbf{M}=\left[\begin{array}{c}
\mathbf{M}_{\mathrm{e}}^{\mathrm{s}} \\
\mathbf{0}
\end{array}\right], \mathbf{f}=\left[\begin{array}{c}
\mathbf{T} \\
\mathbf{Q}
\end{array}\right]
$$

The equilibrium equation of the finite element system is obtained as follows:

$$
\mathbf{Y v}+\mathbf{M}=\mathbf{f}
$$

\subsection{Solution of Equilibrium Equations for Nonlinear System}

The finite difference method is carried out to calculate the equilibrium equation of the nonlinear system. Firstly, the incremental method is used in the time domain, and the time $t$ is discretized into a number of time points, i.e.

$$
0=t_{0}<t_{1}<\ldots<t_{\mathrm{n}}<t_{\mathrm{n}+1}<\ldots<t_{\mathrm{N}}=t
$$

Time increment is expressed as:

$$
\Delta t_{\mathrm{n}+1}=t_{\mathrm{n}+1}-t_{\mathrm{n}}
$$

Complete Lagrange method is adopted, the reference configuration does not change with time. At time $t_{n+1}$ equation (34) can be expressed as follow: 


$$
\mathbf{Y}_{\mathrm{n}+1} \mathbf{v}_{\mathrm{n}+1}+\mathbf{M}_{\mathrm{n}+1}=\mathbf{f}_{\mathrm{n}+1}
$$

By the Newton-Raphson method, the linear form of $\mathbf{M}$ is obtained by the following recursive form:

$$
\mathbf{M}_{\mathrm{n}+1}=\left[\begin{array}{c}
M_{\mathrm{n}+1}^{\mathrm{s}} \\
0
\end{array}\right], \quad M_{\mathrm{n}+1}^{s(i)}=M_{\mathrm{n}+1}^{s(i-1)}+K_{\mathrm{n}+1}^{s(i-1)} \cdot u_{\mathrm{n}+1}^{s(i)}
$$

Where $\boldsymbol{K}_{\mathrm{n}+1}$ is the tangent stiffness matrix of the solid phase, the $\mathbf{0}$ vector is equal to the velocity of the liquid phase or the pressure degree of freedom, $i$ represents the number of iterations.

The recursive relation of iterative displacement is determined by the trapezoidal method:

$$
\Delta u_{\mathrm{n}+1}^{i}=u_{\mathrm{n}+1}^{i}-u_{\mathrm{n}+1}^{i-1}=\omega \Delta t \Delta v_{\mathrm{n}+1}^{i}
$$

Where $\omega$ is the specified time integral parameter, $0 \leq \omega \leq 1$, and when $\omega \geq 1 / 2$, it is an implicit integral, and the equation is unconditionally stable.

The increment of speed is expressed as:

$$
v_{\mathrm{n}+1}^{i}=v_{\mathrm{n}+1}^{i-1}+\Delta v_{\mathrm{n}+1}^{i}
$$

In the $i$ times iteration of speed, equation (34) can be written as follows:

$$
\mathbf{Y}_{\mathrm{n}+1}^{i-1} \mathbf{v}_{\mathrm{n}+1}^{i}+\mathbf{M}_{\mathrm{n}+1}^{i}=\mathbf{f}_{\mathrm{n}+1}^{i-1}
$$

From the above equation can obtain that:

$$
\left(\mathbf{Y}_{\mathrm{n}+1}^{i-1}+\omega \Delta t \mathbf{K}_{\mathrm{n}+1}^{i-1}\right) \Delta v_{\mathrm{n}+1}^{i}=\mathbf{f}_{\mathrm{n}+1}^{i-1}-\mathbf{Y}_{\mathrm{n}+1}^{i-1} \mathbf{v}_{\mathrm{n}+1}^{i-1}-\mathbf{M}_{\mathrm{n}+1}^{i-1}
$$

In the $\Delta t_{\mathrm{n}+1}$ time step, repeated iterative, until the velocity increment $\Delta v_{\mathrm{n}+1}^{i}$ satisfies the convergence criterion. In a time step, the convergence criterion is as follows:

$$
\frac{\left|\Delta v^{i}\right|}{\left|v^{i}\right|}<T O L, \quad T O L=10^{-4}
$$

The solution is updated as the initial value to the next time step when iterative convergence occurs, and repeat iteration until convergence occurs again. Numerical calculation process is compiled in Visual Studio 2012.

\section{Verification and Discussion of the Two Phase Model of Articular Cartilage}

\subsection{Simulation and Validation}

The parameters of hyperelastic model and permeability equation are: $\alpha_{0}=0.1084 \mathrm{Mpa}, \quad \alpha_{1}=0.592 \mathrm{Mpa}, \quad \alpha_{2}=0.0846 \mathrm{Mpa}, \phi_{s 0}=0.2, \kappa_{0}=2.519 \times 10^{-15} \mathrm{~m}^{4} /(\mathrm{Ns}), L=0.0848$. In this study, we took a small piece of long rectangular cartilage model, which is $12 \mathrm{~mm}$ long, $3 \mathrm{~mm}$ wide, and $2 \mathrm{~mm}$ high, and generate volume mesh. Boundary condition is setted as moving in $\mathrm{Y}$ direction, other degrees of freedom are 0 , vertical downward uniform load is imposed at the top of the model, as shown in Figure 1(a), load increment $\Delta F=0.2 \mathrm{~N}$, totally 17 steps are loaded, the stress-strain relationship of cartilage was obtained by simulation, the stress are derived from the second Piola-Kirchhoff stress S, meanwhile the strain is derived from Green strain E. In order to verify the accuracy of the model, 
compared the simulation results with the compressible experimental results of articular cartilage provided by the Department of Orthopedics of the Second Hospital Affiliated of Suzhou University [13], the contrast curve is shown in Figure 1(b). It can be seen from the figure that under the condition of low stress, change rate of the strain is very small, with the increase of stress, the modulus of elasticity is increasing too, this shows that with the increase of stress, the ability to resist elastic deformation is larger. The trend of two curves is coincident, and the stress-strain curve is close to the nonlinear exponential relation, although there is a certain error in the value, but the error range is acceptable.

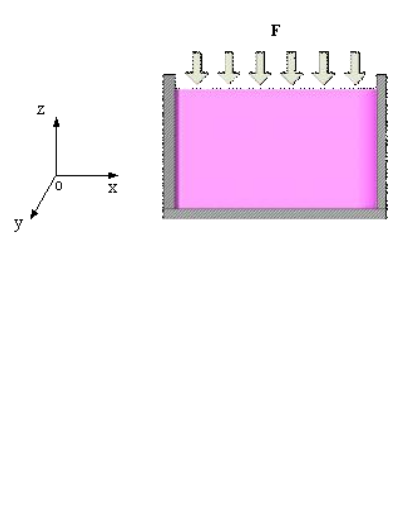

(a) Cartilage compression tests

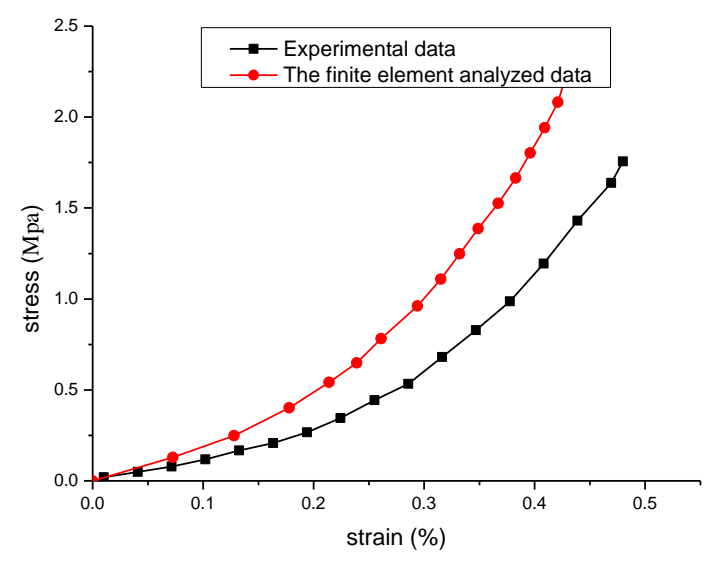

(b) Comparison curves of stress-strain

Figure1. Cartilage Compression Tests and Comparison Curves of StressStrain

For the same piece of articular cartilage model, uniaxial compression experiment was conducted. Placing the cartilage model on the plane, and load $\boldsymbol{F}$ is applied to the top of the model, as shown in Fig. 2(a), per step load increment $\Delta F=0.2 \mathrm{~N}$, the total of 20 steps, and calculate the speed of the highest point of the curve.
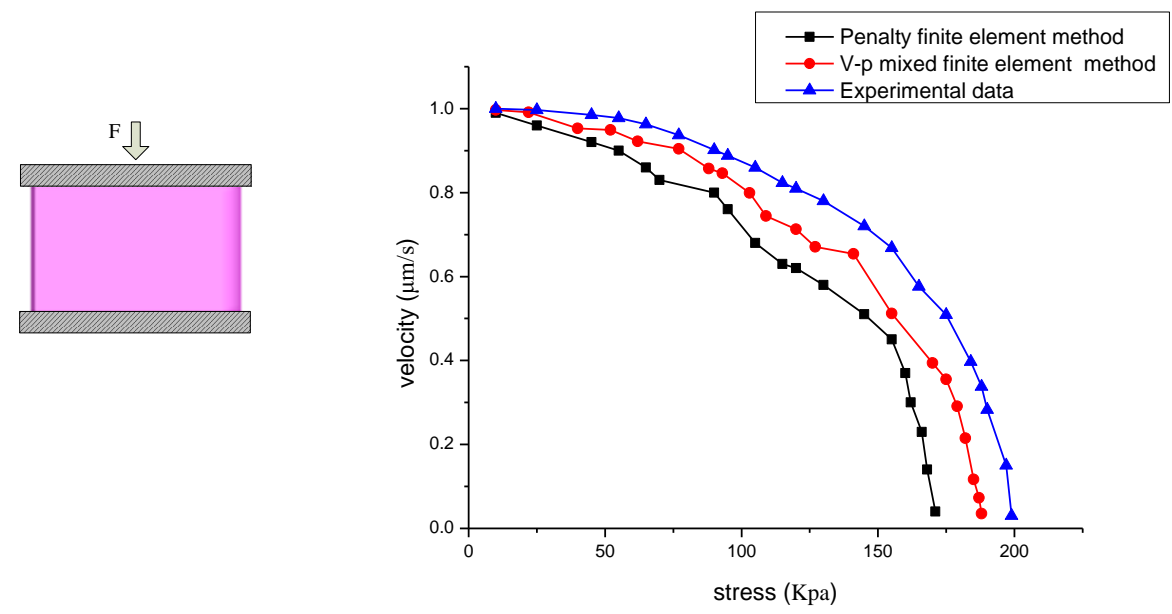

(a) Cartilage uniaxial compression tests

(b) Comparison curves of stress-speed

Figure 2. Uniaxial Compression Tests and Compression Curves of StressSpeed 
The following finite element analysis procedure is carried out within a time step $\Delta t_{1}$, supposing that $\Delta t_{1}=t_{1}-t_{0}=1 \mathrm{~s}$, time integral parameter $\omega=0.6$, each time step reflects the changing process of load at this moment, the relationship between stress and velocity was calculated, as shown in Fig.2 (b). The simulation results are compared with the results that used the penalty finite element method, and the experimental data obtained by the Spilker and Holmes et al. in Rensselaer Polytechnic Institute [14], the contrast curves are shown in Fig.2 (b). It can be seen from the figure that with the increase of the stress, the value of the velocity decreases, i.e. when the pressure on the cartilage surface increases, the ability to resist the external force of cartilage is also increasing, and the rate of deformation is gradually reduced. The overall trend of the three sets of curves is similar. In the initial stage of loading, the results of stress and velocity of three sets have small differences. The error increased with increases in the values of stress. The method proposed in this study is closer to experimental results.

\section{Conclusions}

In this study, based on the hyperelastic solid phase model, a two phase model of articular cartilage was established, and $v-p$ variable control equation of articular cartilage was built up, meanwhile, the finite element equilibrium equation of articular cartilage was established by using the Galerkin weighted residual method. The finite difference method was used to calculate the equilibrium equation of a nonlinear system. The cartilage compression results of experiment and simulation was compared, the accuracy of the proposed model of articular cartilage was verified. Through the finite element simulation of the single axis compression of cartilage, the simulation method was verified.

\section{Acknowledgments}

This research was supported by NSFC (No. 61572159), NCET (NCET-13-0756), Scientific Research Foundation for the Returned Overseas Scholars of Heilongjiang Province and Harbin University of Science and Technology Youth Talent Support Program.

\section{References}

[1] P. Smeriglio, J. H. Lai and L. Dhulipala, "Comparative Potential of Juvenile and Adult Human Articular Chondrocytes for Cartilage Tissue Formation in Three-Dimensional Biomimetic Hydrogels", Tissue Engineering Part A, vol. 3, no. 2, (2014), pp. 75-80.

[2] H. Guo, S. A. Maher and P. A. Torzilli, "A Biphasic Multiscale Study of the Mechanical Microenvironment of Chondrocytes within Articular Cartilage under Unconfined Compression”, Journal of biomechanics, vol. 47, no.11,(2014),pp. 2721-2729.

[3] K. S. Halonen, M. E. Mononen and J. S. Jurvelin, "Deformation of Articular Cartilage During Static Loading of a Knee Joint-Experimental and Finite Element Analysis", Journal of Biomechanics, vol.47, no.10, (2014), pp. 2467-2474.

[4] M. N. Wang, "Modeling and Evaluation to Bone Tissue Based on Cellular Model", Journal of Mechanical Engineering, vol.51, no.11, (2015), pp.142-149.

[5] M. M. Blum and T. C. Ovaert, "Low Friction Hydrogel for Articular Cartilage Repair: Evaluation of Mechanical and Tribological Properties in Comparison with Natural Cartilage Tissue", Materials Science and Engineering: C, vol. 33, no. 7, (2013), pp. 4377-4383.

[6] R. Y. Hori and L. F. Mockros, "Indentation Tests of Human Articular Cartilage", Journal of Biomechanics, vol. 9, no.4, (1976), pp. 259-268.

[7] W. C. Hayes, L. M. Keer and G. Herrmann, "A Mathematical Analysis for Indentation Tests of Articular Cartilage", Journal of Biomechanics, vol. 5, no. 5, (1972), pp. 541-551

[8] V. C. Mow, S. C. Kuei and W. M. Lai, "Biphasic Creep and Stress Relaxation of Articular Cartilage in Compression: Theory and Experiments", Journal of Biomechanical Engineering, vol. 102, no. 1, (1980), pp. 73-84.

[9] V. C. Mow, W. M. Lai and M. H. Holmes, "Advanced Theoretical and Experimental Techniques in 
Cartilage Research”, In Biomechanics: Principles and Applications, vol.23, no.3, (1982), pp. 47-74.

[10] X. Y. Liu, B. Yan and H. J. Liu, "Finite Element Method of Biphasic Porous Medium nonlinear Model of Articular Cartilage”, Chinese Journal of Applied Mechanics, vol. 21, no. 2, (2004), pp. 61-65.

[11] M. H. Holmes and V. C. Mow, "The Nonlinear Characteristics of Soft Gels and Hydrated Connective Tissues in Ultrafiltration”, Journal of Biomechanics, vol.23, no.11, (1990), pp.1145-1156.

[12] M. A. Soltz and G. A. Ateshian, "Experimental Verification and Theoretical Prediction of Cartilage Interstitial Fluid Pressurization at an Impermeable Contact Interface in Confined Compression”, Journal of Biomechanics, vol. 31, no. 10, (1998), pp. 927-934.

[13] W. C. Meng and Q. R. Dong, "Experimental Study on Compression Properties of Articular Cartilage", Journal of Medical Biomechanics, vol.18, no. 1, (2003), pp. 28-33.

[14] J. K. Suh, R. L. Spilker and M. H. Holmes, "A Penalty Finite Element Analysis for Nonlinear Mechanics of Biphasic Hydrated Soft Tissue under Large Deformation", International Journal for Numerical Methods in Engineering, vol. 32, no. 7, (1991), pp. 1411-1439.

\section{Author}

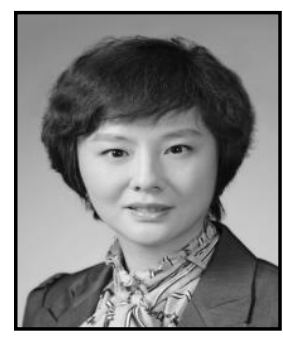

Monan Wang, born in 1973, is currently a Professor at Mechanical \& Power Engineering College, Harbin University of Science and Technology, China. She received her doctor degree from Harbin Engineering University, China, in 2004. Her research interests include medical robots and computer assisted surgery.

Tel: +86-451-86390530; E-mail: qqwmnan@163.com 